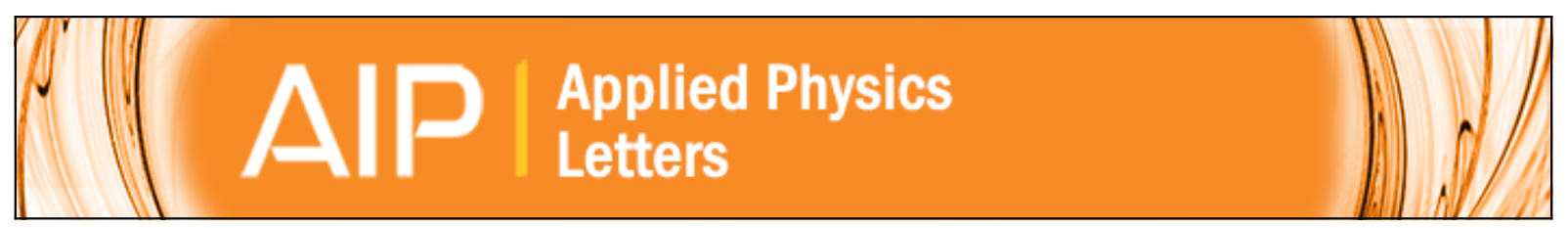

\title{
Controlling spin-orbit interaction in a ferromagnetic Fe/Au double layer
}

Sergey N. Samarin, Oleg M. Artamonov, Alexander P. Baraban, Mikhail Kostylev, Paul Guagliardo, and James F. Williams

Citation: Applied Physics Letters 106, 042404 (2015); doi: 10.1063/1.4906932

View online: http://dx.doi.org/10.1063/1.4906932

View Table of Contents: http://scitation.aip.org/content/aip/journal/apl/106/4?ver=pdfcov

Published by the AIP Publishing

\section{Articles you may be interested in}

A state interaction spin-orbit coupling density matrix renormalization group method

J. Chem. Phys. 144, 234301 (2016); 10.1063/1.4953445

Chiral magnetization textures stabilized by the Dzyaloshinskii-Moriya interaction during spin-orbit torque switching

Appl. Phys. Lett. 104, 092403 (2014); 10.1063/1.4867199

Spin-orbit field switching of magnetization in ferromagnetic films with perpendicular anisotropy

Appl. Phys. Lett. 100, 212405 (2012); 10.1063/1.4722929

Role of exchange interaction and spin-orbit coupling in magnetic domain walls in bulk and thin film Fe J. Appl. Phys. 97, 10A315 (2005); 10.1063/1.1854991

Interlayer exchange coupling in Fe/Au multilayers

J. Appl. Phys. 87, 6588 (2000); 10.1063/1.372779

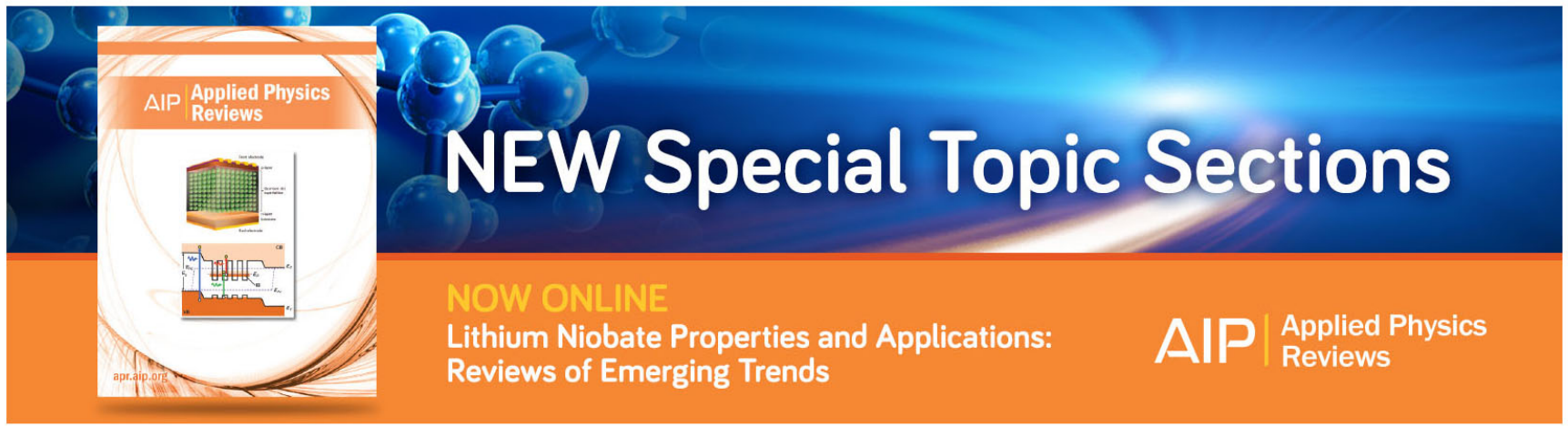




\title{
Controlling spin-orbit interaction in a ferromagnetic Fe/Au double layer
}

\author{
Sergey N. Samarin, ${ }^{1}$ Oleg M. Artamonov, ${ }^{2}$ Alexander P. Baraban, ${ }^{2}$ Mikhail Kostylev, ${ }^{1}$ \\ Paul Guagliardo, ${ }^{3}$ and James F. Williams ${ }^{1}$ \\ ${ }^{1}$ School of Physics, The University of Western Australia, Perth, Western Australia 6009, Australia \\ ${ }^{2}$ Research Institute of Physics, St. Petersburg State University, St. Petersburg 199034, Russia \\ ${ }^{3}$ Centre for Microscopy, Characterisation and Analysis, the University of Western Australia, Perth, \\ Western Australia 6009, Australia
}

(Received 21 December 2014; accepted 18 January 2015; published online 29 January 2015)

\begin{abstract}
Using spin-polarized single- and two-electron spectroscopy, we probe exchange and spin-orbit interaction in a double layer of $\mathrm{Fe}$ and $\mathrm{Au}$ on $\mathrm{W}(110)$ and measure the spin asymmetry of the Bloch spectral density function of the sample. In a $5 \mathrm{ML}$ iron film, the spin-orbit contribution to the measured asymmetry of the (e,2e) spectra was not detectable, whereas a deposition of about $1 \mathrm{ML}$ of gold introduced a substantial spin-orbit component in the measured asymmetry. At the same time, this double layer still exhibits ferromagnetic properties: (i) the spectral density function asymmetry demonstrate imbalance of spin-up and spin-down electron densities in the valence band and (ii) the Stoner excitation asymmetry has almost the same value as in a pure Fe film. (C) 2015 AIP Publishing LLC. [http://dx.doi.org/10.1063/1.4906932]
\end{abstract}

The spin-orbit interaction, i.e., the interaction of an electron spin with the orbital momentum of the electron, is one of the central issues in spintronics. ${ }^{1,2}$ It is responsible for the Spin Hall effect ${ }^{3}$ and determines properties, for example, of topological insulators. ${ }^{4}$ Characterization of those properties follows from measurements of spin-orbit effects and variation of the strength of the spin-orbit interaction (SOI) in a solid structure.

One of the powerful instruments to measure SOI is a magnetic circular dichroism, ${ }^{5}$ which is element specific and provides quantitative information on SOI. The drawback of this technique is that it integrates the results over the Brilloin zone (BZ) and cannot localize the SOI in the momentum space of the valence band. An alternative technique, which is very surface sensitive and localizes SOI in energy-momentum space is Low-Energy Spin-Polarized Two-Electron Spectroscopy (SPe,2e). ${ }^{6-8}$ The essence of two-electron spectroscopy is the detection of two timecorrelated electrons generated by a single incident electron and the measurement of their momenta $\mathbf{k}_{1}$ and $\mathbf{k}_{2}$. The distributions of the correlated electron pairs over the energies of two electrons or over the momenta of the two electrons are called (e,2e) spectra. This technique was previously applied for studying spin-orbit and exchange effects in a thin Co film on W(110). ${ }^{8}$ The spin effects were measured in terms of an asymmetry A, which is defined as the normalized difference of intensities $\mathrm{I}^{+}$and $\mathrm{I}^{-}$, which corresponds to the spectra measured for spin-up incident beam and spindown incident beam, respectively: $\mathrm{A}=\left(\mathrm{I}^{+}-\mathrm{I}^{-}\right) /\left(\mathrm{I}^{+}+\mathrm{I}^{-}\right)$. It was demonstrated that the spin-orbit effect and exchange can be separated in the (e,2e) spectra if corresponding asymmetries are measured for two opposite magnetizations of the sample: M1 and M2 and the polarization vector of the incident beam and the magnetic moment of the sample are collinear. If the asymmetries for reversed and nonreversed sample magnetization are denoted as $\mathrm{A}^{\mathrm{M} 1}$ and $\mathrm{A}^{\mathrm{M} 2}$, the spin-orbit $\left(\mathrm{A}_{\mathrm{SO}}\right)$ and exchange $\left(\mathrm{A}_{\mathrm{ex}}\right)$ contributions are given to leading order by ${ }^{9}$

$$
\begin{gathered}
A_{e x}=1 / 2\left(A^{\mathrm{M} 1}-A^{\mathrm{M} 2}\right), \\
A_{S O}=1 / 2\left(A^{\mathrm{M} 1}+A^{\mathrm{M} 2}\right) .
\end{gathered}
$$

We apply this approach for studying spin-orbit coupling in the valence band of the double layer of iron and gold on $\mathrm{W}(110)$ using spin-polarized two-electron (coincidence pair) spectroscopy.

Details of the spin-polarized two-electron spectrometer and the electron-pair technique are presented elsewhere ${ }^{10}$ and described briefly here. Our spin-polarized electron source is based on a photoemission from a strained GaAs photocathode. ${ }^{11}$ Two micro-channel plate detectors with $75 \mathrm{~mm}$ diameter of active area were used for the electron pair detection. The geometrical arrangement of the experiment is shown in Fig. 1.

The time-of-flight technique was applied for the electron energy measurements. ${ }^{12}$ The incident electron beam was pulsed to have a reference point on the time scale. Energy conservation for the $(\mathrm{e}, 2 \mathrm{e})$ reaction implies that $\mathrm{E}_{\mathrm{b}}=\left(\mathrm{E}_{1}+\mathrm{E}_{2}\right)-\mathrm{E}_{\mathrm{o}}$, where

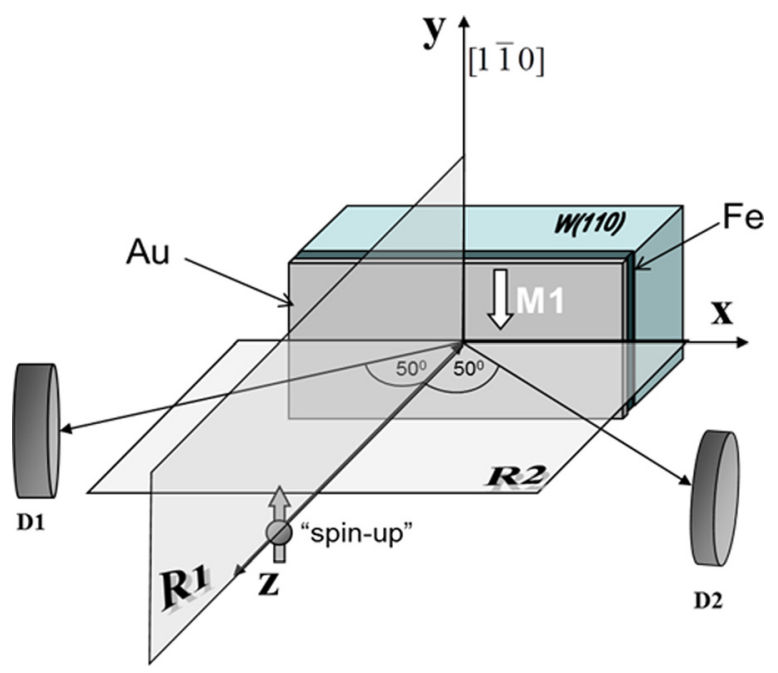

FIG. 1. Spin-polarized two-electron scattering geometry. 

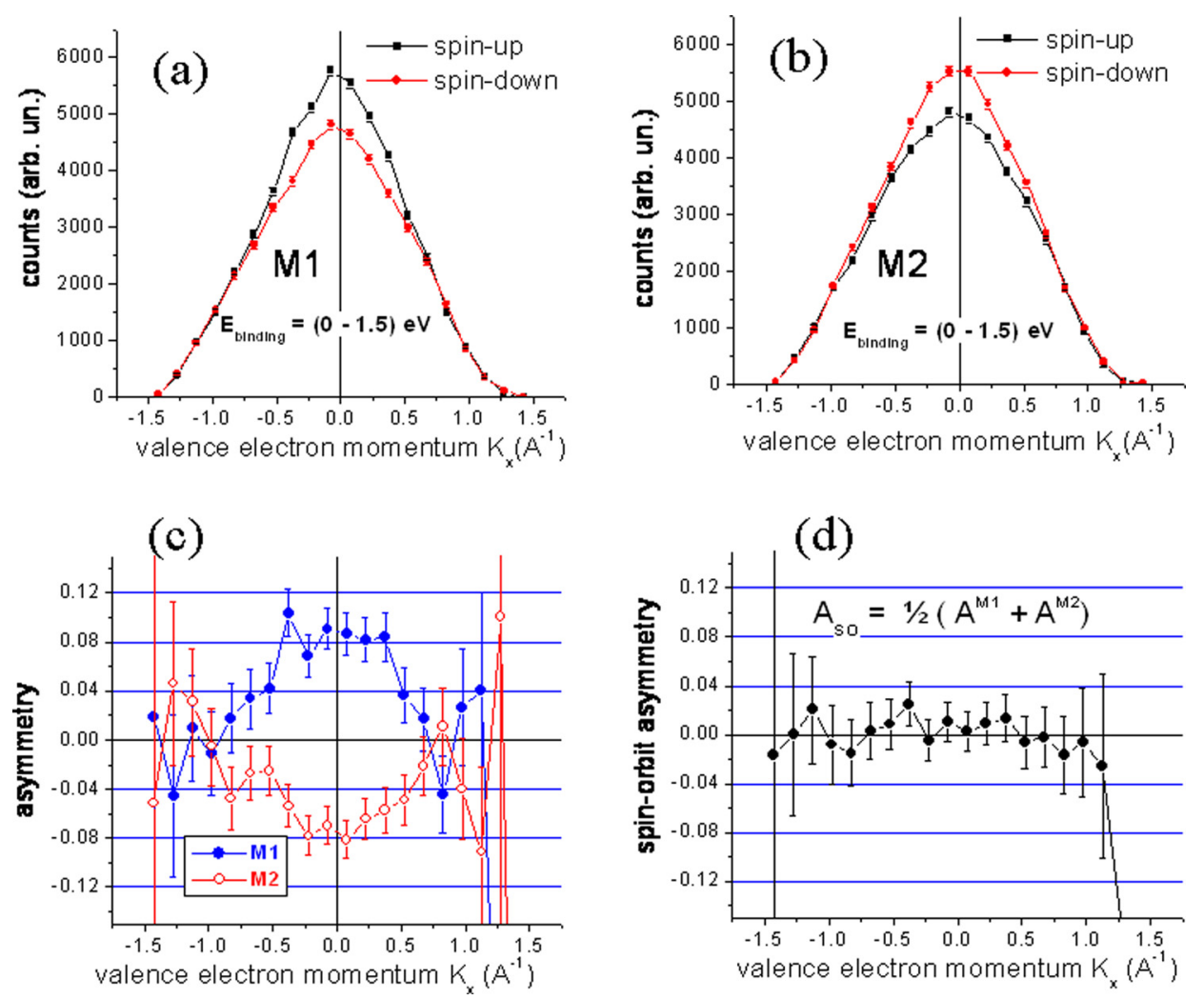

FIG. 2. $\mathrm{K}_{\mathrm{x}}$-distributions measured with spin-up and spin down incident electrons for magnetization M1 (a) and magnetization M2 (b); (c) asymmetries of $\mathrm{K}_{\mathrm{x}}$-distributions for M1 and M2; and (d) spin-orbit component of asymmetry.
$E_{b}$ is the binding energy of the valence electron, $E_{o}$ is the primary electron energy, and $E_{1}$ and $E_{2}$ are the energies of the two detected correlated electrons. For these pairs of electrons, the parallel-to-the-surface component of the total momentum is conserved: $\mathbf{k}_{\mathrm{b} \|}=\mathbf{k}_{1 \|}+\mathbf{k}_{2 \|}-\mathbf{k}_{0 \|}+\mathbf{G}$, where $\mathbf{k}_{\mathrm{b}}$ is the valence electron momentum, $\mathbf{k}_{1}$ and $\mathbf{k}_{2}$ are momenta of detected electrons, $\mathbf{k}_{0}$ is the incident electron momentum, and $\mathbf{G}$ is the reciprocal lattice vector. Thus, energy and momentum conservation allows the valence electron involved in the collision to be localized in energy-momentum space in contrast to a single-electron spectroscopy (EELS), where the result of a scattering event is integrated over the unresolved state of the second electron.

Prior to deposition of the films, the substrate W(110) was thoroughly cleaned using the well established routine ${ }^{13,14}$ including oxygen treatment of the surface followed by high temperature flashes. The 5 monolayers (ML) of Fe film was deposited using Omicron commercial evaporator EFM-3. A thin (about $1 \mathrm{ML}$ ) Au film was deposited by evaporating a small piece of gold from $\mathrm{v}$-shaped $0.2 \mathrm{~mm}$ diameter tungsten wire by resistive heating. The thickness of the films was estimated by Auger electron spectroscopy and a quartz microbalance.

First, we checked the exchange and spin-orbit components of the asymmetry of (SPe,2e) spectra of a pure Fe film on W(110). We expected to see, in analogy with studies of a Co film, ${ }^{8}$ a substantial contribution of the spin-orbit asymmetry in addition to the exchange effect in a ferromagnetic film. On the contrary, there was no detectable spin-orbit component in the measured asymmetry of the (SPe,2e) spectra. Fig. 2 shows $\mathrm{K}_{\mathrm{x}}$-distributions measured with spin-up and spin-down incident electrons for two opposite magnetizations of the sample. Each of the distributions presents the number of correlated electron pairs as a function of the $\mathrm{x}$-component of the valence electron momentum within the binding energy band of $1.5 \mathrm{eV}$ just below the Fermi energy. Figs. 2(a) and 2(b) indicate that $\mathrm{K}_{\mathrm{x}}$-distributions are very symmetric with respect to the point $\mathrm{K}_{\mathrm{x}}=0$, which is the centre of the Brilloin zone. The maximum difference between spin-up spectrum and spin-down spectrum observed at the centre of the $\mathrm{BZ}$ and this difference changes the sign, when the magnetization of the sample reverses. This is an indication that the difference in intensity is due to the exchange effect. ${ }^{15,16}$ Fig. 2(c) shows asymmetries of $\mathrm{K}_{\mathrm{x}}$-distributions for M1 and M2 magnetizations of the sample. The spin-orbit component of the asymmetry was calculated as indicated above according to Eq. (2). It is seen from Fig. 2(d) that it is zero within the error bars.

When a thin layer of gold was deposited, as confirmed by Auger electron spectroscopy (Fig. 3), the double layer of $\mathrm{Au} / \mathrm{Fe}$ was still exhibiting ferromagnetic properties. Indeed

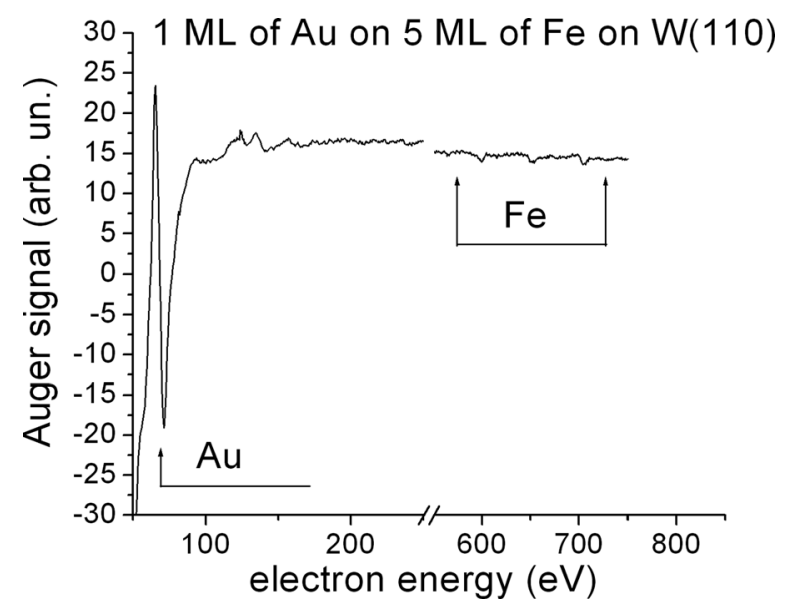

FIG. 3. Auger electron spectrum of $\mathrm{Au} / \mathrm{Fe}$ double layer. 

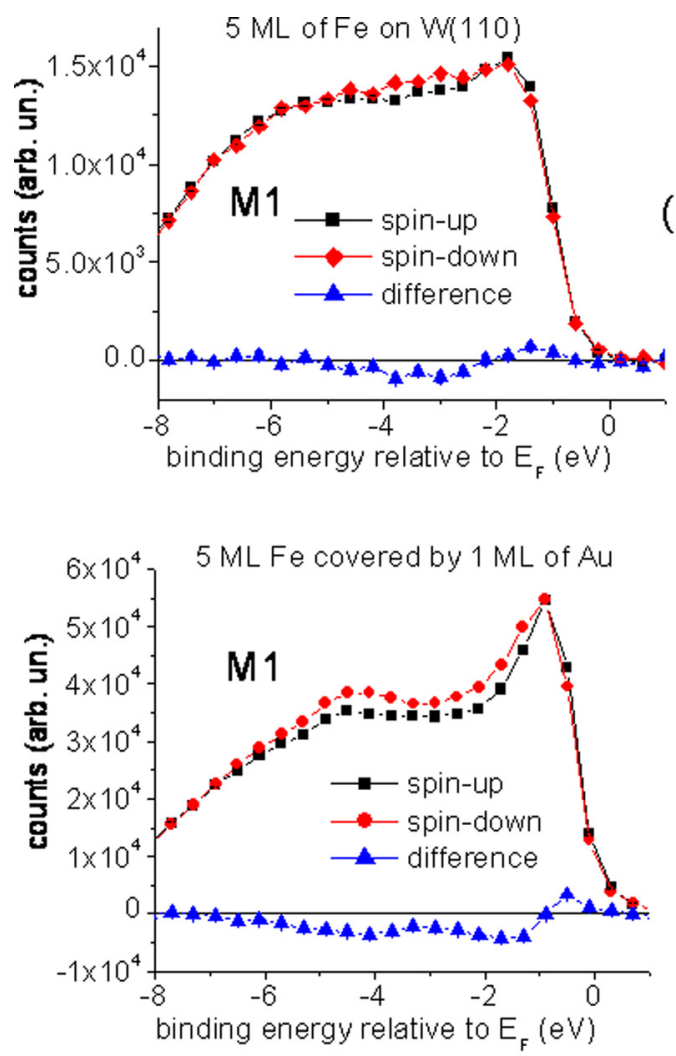

(b)

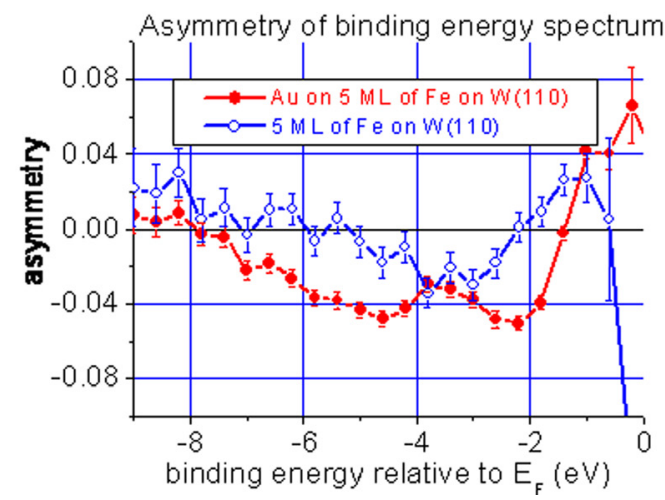

(c)

FIG. 4. Binding energy spectra for Fe film (a) and for Fe film covered by a layer of $\mathrm{Au}(\mathrm{b})$; and (c) asymmetries of binding energy spectra for Fe film and $\mathrm{Au} / \mathrm{Fe}$ bilayer.

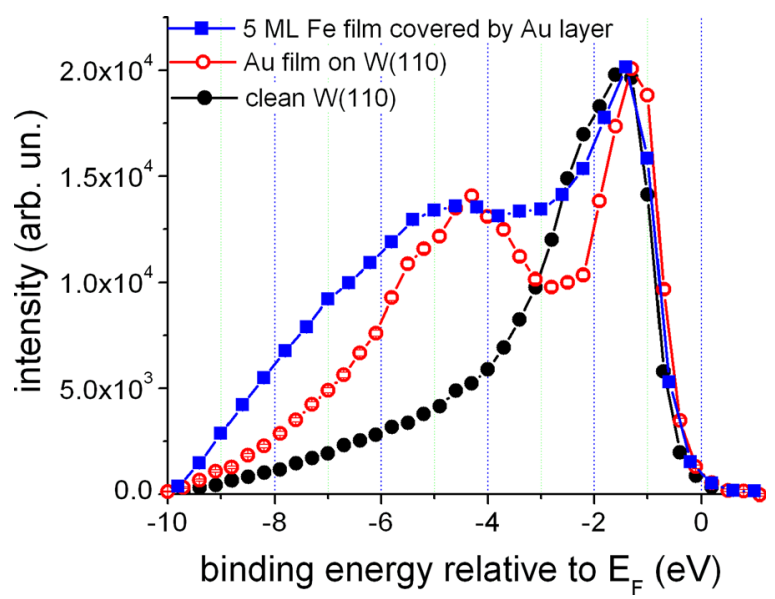

FIG. 5. Spin integrated binding energy spectra of W(110), Au film on W(110) and Fe film covered by Au layer. the binding energy spectra recorded with spin-up and spindown incident electrons show imbalance of spin-up and spin-down states in the valence band of the sample (Fig. 4). Comparison of the binding energy asymmetry spectra of pure Fe film and of the Fe film with the gold cap layer shows that the shape of the asymmetry is different in these two cases (Fig. 4(c)). Moreover, the asymmetry spectrum of a double layer of $\mathrm{Au} / \mathrm{Fe}$ shows a larger value and a larger energy range of asymmetry (Fig. 4(c)).

The change of asymmetry in the binding energy spectrum after an Au layer deposition indicates the modification of the electronic structure of the surface. It also suggests that a thin gold layer may become ferromagnetic. In fact, there are a number of indications that the gold exhibits ferromagnetic properties when its shape indicates nanoparticles ${ }^{17}$ or thin layers. ${ }^{18}$ However, the shape of the binding energy spectrum of a double layer of $\mathrm{Au} / \mathrm{Fe}$ is different from the spectrum of an Fe film on W(110) and from the spectrum of a $\mathrm{Au}$ layer on W(110) (Fig. 5).

That result confirms the suggestion that the surface electronic structure of the double layer of $\mathrm{Au} / \mathrm{Fe}$ is modified due to hybridization at the interface of the Au-Fe states. In addition, a strong exchange field of the Fe film can induce a magnetic moment in the Au film; hence, an imbalance of spin-up and spin-down states in the valence band. This imbalance shows up as an asymmetry in the binding energy spectrum of the double layer (Fig. 4(c)). The possibility of induced spin polarization in a nonmagnetic layer by an adjacent ferromagnetic layer was demonstrated in Ref. 19.

We analyse now the $\mathrm{K}_{\mathrm{x}}$-distributions and corresponding asymmetries for the $\mathrm{Au} / \mathrm{Fe}$ double layer. We chose two binding energy bands where the $\mathrm{K}_{\mathrm{x}}$-distributions were measured

\section{$5 \mathrm{ML}$ of Fe covered by $1 \mathrm{ML}$ of $\mathrm{Au}$}

\section{Binding energy: $(-2 \div-1) \mathrm{eV}$ Binding energy: $(-1 \div 0) \mathrm{eV}$}

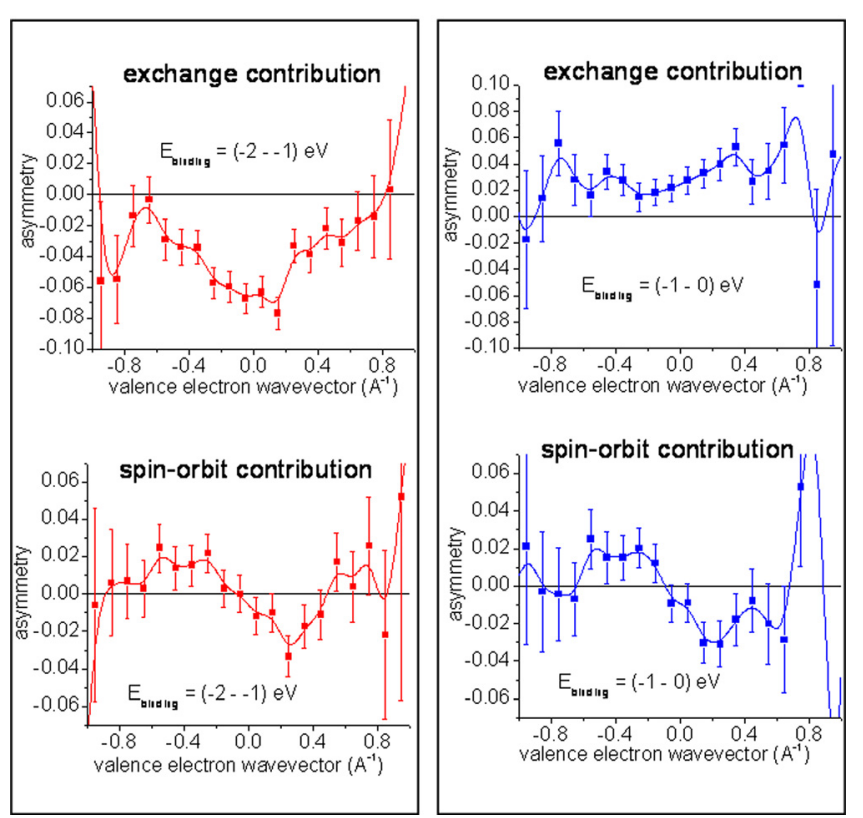

FIG. 6. Asymmetries of $\mathrm{K}_{\mathrm{x}}$-distributions measure within two different binding energy bands (left and right panels): $(-2 \div-1) \mathrm{eV}$ and $(-1 \div 0) \mathrm{eV}$, respectively. 
with spin-up and spin-down incident electrons. The first band spans from 0 down to $-1 \mathrm{eV}$ binding energy, where the difference and asymmetry of the binding energy spectrum are positive (Fig. 4(b)) and the second band from $-1 \mathrm{eV}$ down to $-2 \mathrm{eV}$ binding energy where the difference and asymmetry are negative. Fig. 6 shows the exchange and spin-orbit contributions to the asymmetries of the $\mathrm{K}_{\mathrm{x}}$ distributions measured within the above binding energy bands. The left column of Fig. 6 represents the exchange and spin-orbit asymmetries of the $\mathrm{K}_{\mathrm{x}}$-distributions within the binding energy from $-1 \mathrm{eV}$ to $-2 \mathrm{eV}$. The right column shows corresponding asymmetries within the binding energy band from 0 to $-1 \mathrm{eV}$. In both cases, there are the contributions of exchange and spin-orbit asymmetries.

The latter is very similar in shape in two energy bands and reaches value up to $2 \%$. In contrast, the exchange component of asymmetry, in the binding energy band just below the Fermi energy, is almost flat on average and reaches the value of about $3 \%$, whereas in the binding energy band from $-1 \mathrm{eV}$ to $-2 \mathrm{eV}$ the exchange component has a negative maximum in the middle and reaches the value of about $7 \%$. The most important conclusion of this analysis is that the deposition of a very thin layer of gold introduces a substantial spin-orbit interaction on the surface of the $\mathrm{Au} / \mathrm{Fe}$ double-layer, which retains ferromagnetic properties. The signature of ferromagnetism is the exchange asymmetry in the $\mathrm{K}_{\mathrm{x}}$-distributions as well as the asymmetry in the binding energy spectrum. In addition, we measured electron energy loss spectra of the $\mathrm{Au} / \mathrm{Fe}$ system for two opposite polarizations of the incident beam and found non-zero Stoner excitation asymmetry, which is indicative of a ferromagnetic surface. ${ }^{9}$ This asymmetry was also measured two months after the gold layer deposition and showed the same value as just after the deposition and so demonstrated a stable ferromagnetic surface protected against vacuum contamination. These findings are in line with the results of Ref. 18, where magnetic-circular dichroism was applied to reveal that the orbital magnetic moment of the constituent $\mathrm{Fe}$ atoms in the bcc $\mathrm{Fe}-\mathrm{Au}$ alloy film is about twice larger than that of pure $\mathrm{Fe}$. It was suggested that alloy-like regions are formed at the interface of the $\mathrm{Au} / \mathrm{Fe}$ bilayer structure. This implies a hybridization of the electronic states of $\mathrm{Au}$ and $\mathrm{Fe}$ and probably enhanced the magnetic moment of $\mathrm{Fe}$ (and/or an induced magnetic moment of $\mathrm{Au}){ }^{18}$

This research was supported by the Australian Research Council and the University of Western Australia. OMA acknowledges Saint-Petersburg State University for a Research Grant 11.38.187.2014. We gratefully acknowledge fruitful discussions with J. Kirschner and F. O. Schumann. We thank S. Key and G. Light (UWA) for their technical support.

${ }^{1}$ S. A. Wolf, D. D. Awschalom, R. A. Buhrman, J. M. Daughton, S. von Molnàr, M. L. Roukes, A. Y. Chtchelkanova, and D. M. Treger, Science 294, 1488 (2001).

${ }^{2}$ I. Žutć, J. Fabian, and S. D. Sarma, Rev. Mod. Phys. 76, 323 (2004).

${ }^{3}$ M. I. Dyakonov and V. I. Perel, Phys. Lett. 35A, 459 (1971).

${ }^{4}$ J. E. Moore, Nature 464, 194 (2010).

${ }^{5}$ J. Stöhr, J. Magn. Magn. Mater. 200, 470 (1999).

${ }^{6}$ S. Samarin, O. M. Artamonov, V. N. Petrov, M. Kostylev, L. Pravica, A. Baraban, and J. F. Williams, Phys. Rev. B 84, 184433 (2011).

${ }^{7}$ S. Samarin, O. M. Artamonov, A. D. Sergeant, J. Kirschner, and J. F. Williams, J. Phys.: Conf. Ser. 100, 072033 (2008).

${ }^{8}$ S. N. Samarin, O. M. Artamonov, A. D. Sergeant, and J. F. Williams, Surf. Sci. 601, 4343 (2007).

${ }^{9}$ J. Kirschner, "Polarized electrons at surfaces," in Springer Tracts in Modern Physics (Springer, Heidelberg, 1985), Vol. 106.

${ }^{10}$ S. Samarin, J. Berakdar, A. Suvorova, O. M. Artamonov, D. K. Waterhouse, J. Kirschner, and J. F. Williams, Surf. Sci. 548, 187 (2004).

${ }^{11}$ D. T. Pierce, R. J. Celotta, G.-C. Wang, W. N. Unertl, A. Galejs, C. E. Kuyatt, and S. R. Mielczarek, Rev. Sci. Instrum. 51, 478 (1980).

${ }^{12}$ S. N. Samarin, O. M. Artamonov, D. K. Waterhouse, J. Kirschner, A. Morozov, and J. F. Williams, Rev. Sci. Instrum. 74(3), 1274 (2003).

${ }^{13}$ K. Zakeri, T. R. F. Peixoto, Y. Zhang, J. Prokop, and J. Kirschner, Surf. Sci. 604, L1 (2010).

${ }^{14}$ R. Cortenraada, S. N. Ermolov, V. N. Semenov, A. W. Denier van der Gon, V. G. Glebovsky, S. I. Bozhko, and H. H. Brongersma, J. Cryst. Growth 222, 154 (2001).

${ }^{15}$ S. N. Samarin, J. Berakdar, O. Artamonov, and J. Kirschner, Phys. Rev. Lett. 85, 1746 (2000).

${ }^{16}$ S. Samarin, O. Artamonov, J. Berakdar, A. Morozov, and J. Kirschner, Surf. Sci. 482(2), 1015 (2001).

${ }^{17}$ Y. Yamamoto, T. Miura, M. Suzuki, N. Kawamura, H. Miyagawa, T. Nakamura, K. Kobayashi, T. Teranishi, and H. Hori, Phys. Rev. Lett. 93, 116801-1 (2004).

${ }^{18}$ K. W. Kim, Y. H. Hyun, R. Gontarz, Y. V. Kudryavtsev, and Y. P. Lee, Phys. Status Solidi A 196(1), 197-200 (2003).

${ }^{19}$ M. G. Samant, J. Stöhr, S. S. P. Parkin, G. A. Held, B. D. Hermsmeier, F. Herman, M. van Schilfgaarde, L.-C. Duda, D. C. Mancini, N. Wassdahl, and R. Nakajima, Phys. Rev. Lett. 72, 1112 (1994). 